BMJ Open

Diabetes

Research

\& Care

\title{
Process quality of diabetes care under favorable access to healthcare: a 2-year longitudinal study using claims data in Japan
}

\author{
Hirokazu Tanaka, ${ }^{1}$ Jun Tomio, ${ }^{1}$ Takehiro Sugiyama, ${ }^{1,2}$ Yasuki Kobayashi ${ }^{1}$
}

To cite: Tanaka H, Tomio J, Sugiyama $\mathrm{T}$, et al. Process quality of diabetes care under favorable access to healthcare: a 2-year longitudinal study using claims data in Japan. BMJ Open Diabetes Research and Care 2016;4:e000291. doi:10.1136/bmjdrc-2016000291

Received 30 June 2016 Revised 12 August 2016 Accepted 21 August 2016

CrossMark

${ }^{1}$ Department of Public Health, Graduate School of Medicine, The University of Tokyo, Tokyo, Japan

${ }^{2}$ Diabetes and Metabolism Information Center, Research Institute, National Center for Global Health and Medicine, Tokyo, Japan

Correspondence to Dr Yasuki Kobayashi; yasukik@m.u-tokyo.ac.jp

\section{ABSTRACT}

Objective: To investigate the process quality of diabetes care provided to patients under universal health insurance coverage.

Research design and methods: Using claim data for 570363 beneficiaries aged 20-69 years who were covered by Health Insurance Societies between April 2010 and March 2012, we identified patients with type 2 diabetes who made follow-up visits at least every 3 months in the first year (subjectidentification year). We assessed patient adherence to follow-up visits in the second year (qualityreporting year), calculated the proportion of patients that completed routine examinations for glycemic control and complications, and evaluated associations between characteristics of patients and quality indicators using multivariable logistic regression models.

Results: We identified 12909 patients with diabetes; in the subject-identification year, 1415 $(11.0 \%)$ had prescriptions for insulin injections, $6049(46.9 \%)$ had prescriptions for oral antihyperglycemic agents, and $5445(42.2 \%)$ had no diabetes-related prescriptions. Among patients using medication, 474 (6.4\%) dropped out in the qualityreporting year. The adjusted percentages of quality indicators among patients using oral antihyperglycemic agents were $95.8 \%$ for glycated hemoglobin, $35.6 \%$ for eye examinations, $15.4 \%$ for urine microalbumin excretion, and $90.6 \%$ for serum lipids; the percentages among patients taking insulin were the same or higher. Annual testing for glycated hemoglobin was less frequent in patients aged $40-$ 49 years than in patients aged $60-69$ years (OR 0.77 ; $95 \% \mathrm{Cl} 0.66$ to 0.91 ). Fewer men than women (OR $0.59 ; 95 \% \mathrm{Cl} 0.54$ to 0.64 ), and fewer patients aged 40-49 years than those aged 60-69 years (OR 0.56 ; $95 \% \mathrm{Cl} 0.50$ to 0.63 ) tended to complete routine eye examinations.

Conclusions: Screening for retinopathy and nephropathy was less frequent than required despite favorable conditions for access to healthcare in Japan. Suboptimal quality of care appeared to depend on provider factors as well as patient factors, such as limited access to retinopathy prevention among working-age men with diabetes.

\section{Key messages}

- How optimal is the process quality of diabetes care provided to the patients under universal health insurance coverage with a generous benefits package in Japan?

- Although examinations for glycemic control and lipid profile monitoring were frequent, screening for retinopathy and nephropathy was less frequent than recommended in clinical guidelines.

- The suboptimal quality of care might be attributable to healthcare provider factors as well as patient factors such as low access to retinopathy prevention among working-age men with diabetes.

\section{INTRODUCTIONS}

Diabetes is one of the most common and costly medical conditions; reducing the disease and its economic burden has become a universal goal in medical care and health policy throughout the world. ${ }^{1}$ There is evidence that high-quality care and attending physicians regularly reduce the risk of microvascular and macrovascular disease complications and consequently, mortality rates. $^{2-4}$

The quality of diabetes care can be measured by process measurements including regular glycated hemoglobin (HbA1c) tests, as well as intermediate outcome measures such as achievement of glycemic control. ${ }^{5}$ Previous studies suggest that patient and healthcare provider factors influence the overall quality of diabetes care. ${ }^{6}$ Patient factors including access to care influence the quality of diabetes care. ${ }^{7}$ Some previous studies mention that patient characteristics and comorbid conditions affect the quality of diabetes care, ${ }^{8} 9$ whereas one study shows that women are less likely to be monitored for eye complications. ${ }^{10}$ In addition, dropout from care (referred to as 'dropout' or 'the dropout patients' hereafter) is a problematic 
issue in diabetes care. ${ }^{11}{ }^{12}$ Dropouts have no access to care, which leads to poor glycemic control through the lack of proper medical care, especially lack of medications. ${ }^{3}$ Among healthcare provider factors, physicians' beliefs, attitude, and knowledge of diabetes care affect glycemic control and clinical practice directly. ${ }^{13}$ Clinical guidelines for diabetes from many organizations recommend regular HbA1c tests, retinopathy screening, nephropathy screening, and lipid profile monitoring. ${ }^{14-}$

${ }^{16}$ Previous studies of process measurement and adherence to clinical guidelines have been reported in the USA, ${ }^{17}{ }^{18}$ Canada, $^{19}$ Europe $^{20}{ }^{21}$ and elsewhere. ${ }^{22-24}$ These studies show that the 'evidence-practice gap' among healthcare providers is still a main barrier to the quality of diabetes care, although the gap is gradually decreasing. ${ }^{17} 2123$

In Japan, patients' access to the health service is generally high because of universal health insurance coverage and a generous benefits package including unrestricted access to almost any provider. ${ }^{25}$ Therefore, it can provide favorable conditions for regular testing of glycemic control as well as screening of complications in chronic diseases such as diabetes. While diabetes is quite prevalent in Japan (7.2 million adults suffer diabetes, ranked 10th in the world), ${ }^{1}$ improvement of diabetes care has been a growing challenge among Japan's health service sectors. ${ }^{26}$ Previous studies have been limited to the elderly population ${ }^{27}$ and physicians' performance in a single hospital in Japan. ${ }^{28}$ Moreover, these studies failed to include both dropout patients and healthcare provider factors.

Within this context, this study aimed to assess the process quality of diabetes care in terms of persistent follow-up and routine examinations of glycemic control and other complications under favorable access to healthcare conditions using health insurance claims data.

\section{RESEARCH DESIGN AND METHODS Study design}

We conducted a 2-year longitudinal observational study using health insurance claims data. We defined the first fiscal year (April 2010 to March 2011) as the subject-identification year. Subsequently, we observed whether the patients made follow-up visits or dropped out in the second fiscal year (April 2011 to March 2012, the quality-reporting year). Finally, we collected data on examinations for glycemic control and diabetes complications among those who were followed up in the quality-reporting year. The Research Ethics Committee of the Graduate School of Medicine, the University of Tokyo approved this study after due ethical consideration (approval number 2917-(6)).

\section{Data sources}

We analyzed health insurance claims data managed by the Japan Medical Data Center (JMDC) Claims
Database. ${ }^{29}$ Japan has a universal healthcare system; all residents must enrol in a health insurance program that is uniquely determined by their age, residential address, and profession. ${ }^{25}$ For example, 'Society-managed Health Insurance' is health insurance for workers in large companies and factories and their dependents, and is managed by the Health Insurance Society. ${ }^{30}$ Premiums are calculated based on the beneficiaries' monthly income. Beneficiaries pay $30 \%$ of inpatient or outpatient costs as copayment (beneficiaries aged 75 years and over pay $10 \%$ ), up to the threshold amount of catastrophic coverage. The JMDC Claims Database contains claims data from several Health Insurance Societies for which standardized disease classifications and anonymous record linkage have been developed since 2003. ${ }^{29}$ Recently, this database has been used for research on several different topics, including diabetes care. ${ }^{31-36}$

The JMDC Claims Database held data for 570363 beneficiaries (320 146 men, 250217 women) aged 20-69 years who were covered by Health Insurance Societies between April 2010 and March 2012; this became our study population. We extracted data for beneficiaries with at least one medical claim for diabetes excluding insulin-dependent diabetes (E11-14, International Statistical Classification of Diseases and Related Health Problems, 10th Revision (ICD-10)), or at least one prescription for an injection (insulin or glucagon-like peptide-1 (GLP-1) analog) or oral antihyperglycemic agents during the study period.

\section{Identification of patients with type 2 diabetes and dropouts}

In Japan's medical claims system diagnoses of 'diabetes' must be recorded on the claim forms to allow reimbursements for diagnostic tests and treatment. Hence, diagnosis data from claim forms have high sensitivity. However, some of the diagnoses are recorded only for reimbursing diagnostic tests for diabetes and hence are not necessarily accurate. Thus, in order to increase the specificity of case detection, we additionally classified patients with diabetes into three groups based on prescriptions during the subject-identification year, as follows: insulin injection (at least one prescription of insulin products), oral antihyperglycemic agents (prescribed for 28 days or more), and no diabetes-related prescriptions. Insulin products were included as 'A10C' and 'A10D' in the Anatomical Therapeutic Chemical (ATC) classification system managed by the WHO. ${ }^{37}$ Oral antihyperglycemic agents were included as 'A10H', 'A10J', 'A10K', 'A10L', 'A10M', 'A10N', and 'A10S' in the ATC classification system. We excluded 'voglibose, $0.2 \mathrm{mg}$ (A10L) prescriptions because these were also under insurance coverage for prevention of type 2 diabetes. We excluded diagnoses of 'suspected' diabetes in the claim forms. A flow diagram showing how we identified patients with type 2 diabetes is shown in figure 1.

We identified patients with type 2 diabetes who had regularly visited clinics or hospitals at least every 3 months, regardless of whether or not they were 


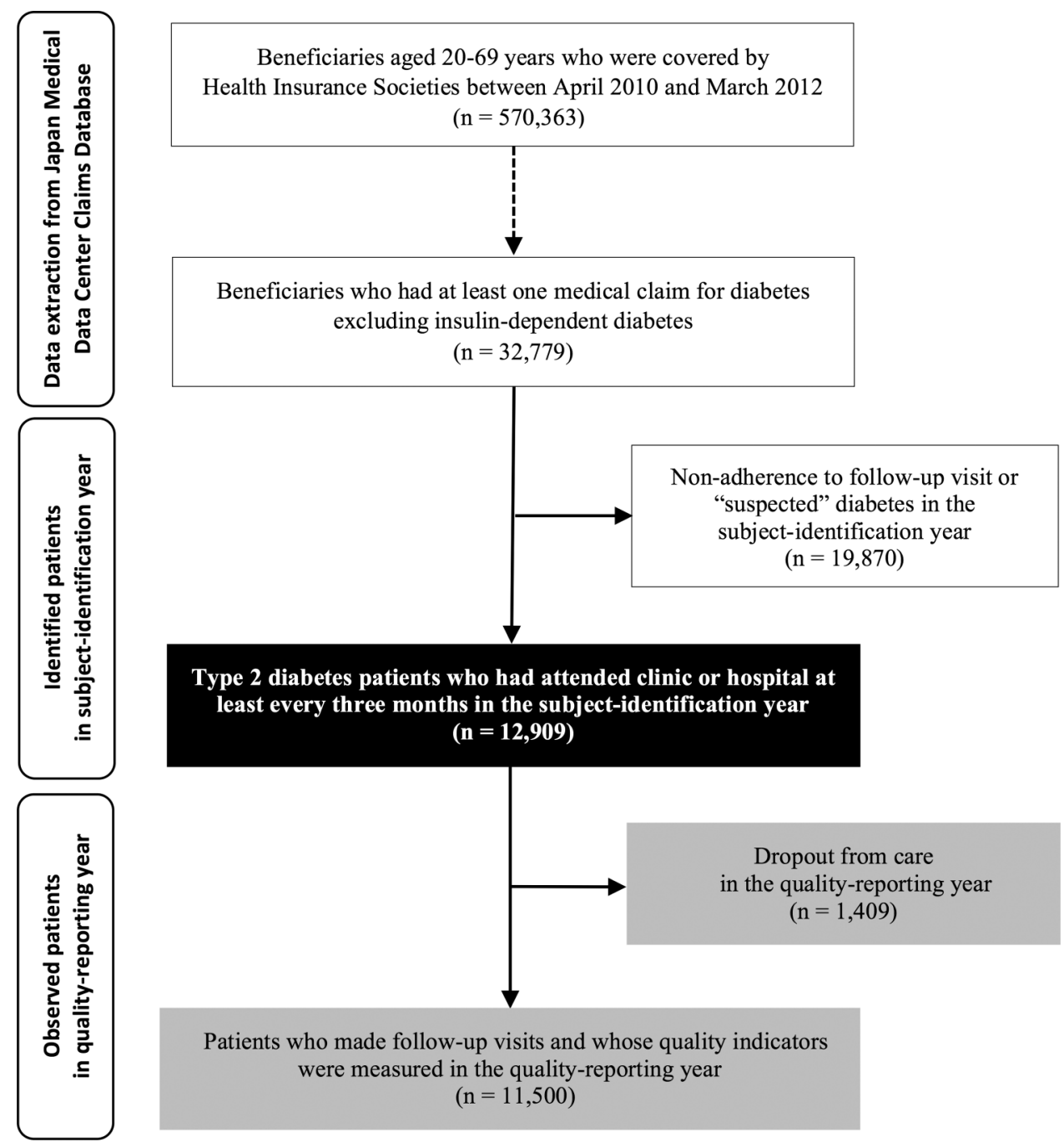

Figure 1 Flow diagram to identify patients with type 2 diabetes.

hospitalized, in the subject-identification year. This definition of adherence to follow-up visit was recommended as a quality indicator in a previous study. ${ }^{38}$ We defined non-adherence to follow-up visits (no visits to the clinic or hospital for 3 months or more) in the quality-reporting year as a 'dropout'. We assessed inpatient as well as outpatient claims records in the quality-reporting year to identify whether patients adhered to follow-up; we also evaluated the number of tests performed during hospitalization.

\section{Quality indicators}

We evaluated the following four key aspects of the process quality of diabetes care: glycemic control, retinopathy screening, nephropathy screening, and lipid profile monitoring. These indicators were evaluated for patients who made follow-up visits in the quality-reporting year.

An HbAlc test at least every year $(\geq 1$ per year) or every 3 months ( $\geq 1$ per 3 months) was defined as a quality indicator for glycemic control based on previous studies. ${ }^{14} 152738$ Eye examinations included complete fundus examinations, pan-vitreoretinal examinations, and/or fundus cameras. ${ }^{27}$ For retinopathy screening, the Japan Diabetes Study (JDS) guideline recommending eye examinations at the diagnosis of diabetes and subsequent annual follow-ups was accepted. ${ }^{14}$ Two indicators for nephropathy screening were defined: one or more urine microalbumin excretion tests (as recommended by the JDS guidelines) and serum creatinine tests. ${ }^{142738}$ The serum lipid test rate (any 1 among total cholesterol, low-density lipoprotein cholesterol, highdensity lipoprotein cholesterol, and triglyceride tests) was also calculated to assess lipid profile monitoring. An annual serum lipid test is recommended by several clinical guidelines but the JDS guidelines do not mention a specific frequency of lipid profile monitoring. ${ }^{14} 38$

\section{Patient characteristics and types of medical consultation facility}

For patient characteristics, we used sex and age in 2010 as estimated from the beneficiaries' birth year. We 
diagnosed comorbid conditions if patients had two or more medical claims for other diseases in the subject-identification year. We chose nine comorbid conditions as follows: ${ }^{9}{ }^{27}$ hyperlipidemia (E78, ICD-10), hypertension (I10-15), chronic respiratory disease (J40-47), kidney and vesicoureteral disorders (N00-16, 20-39), cerebral vascular disorder (I60-69), cancer (C00-99, D00-48), ischemic heart disease (I20-25), mental disorders (F20-39), and renal failure (N17-19). In order to investigate the relationship between types of medical consultation facilities and process quality indicators, we categorized the patients into the following two categories in terms of consultation patterns in the subject-identification year: patients who visited only one clinic (medical facility for outpatients only or with $\leq 19$ beds) and others (those who visited multiple facilities or only 1 hospital).

\section{Statistical analysis}

Age was categorized into five groups: 20-29, 30-39, 40$49,50-59$, and 60-69 years. We calculated sex-specific and age-specific proportions of patients with diabetes in the subject-identification year.

We computed the proportion of dropouts in the quality-reporting year to assess the dropout rate per year. After the dropouts were excluded, we performed separate multivariate logistic regression analyses for six quality indicators adjusted for the covariates (sex, age, prescription, comorbid conditions, and type of medical consultation facility). To estimate the quality indicator percentages after adjustment by covariates (sex, age, comorbid conditions, type of medical consultation facility conditions), we calculated adjusted percentages by prescription. For the urine microalbumin excretion test and serum creatinine test models, we excluded 1413 individuals with known kidney and vesicoureteral disorders or renal failure, for whom urine microalbuminuria screening was no longer recommended.

We used Stata V.13.1 (StataCorp, College Station, Texas, USA) for the statistical analysis and data management. A $p$ value of $<0.05$ was considered to be significant.

\section{RESULTS}

\section{Patient characteristics}

Patient characteristics are shown in table 1 . We identified 12909 patients with type 2 diabetes $(2.26 \%$ of the study population) who made the required follow-up visits in the subject-identification year. The proportion of patients with diabetes increased with age for both men and women; $0.13 \%$ of men and $0.17 \%$ of women aged $20-29$, and $17.0 \%$ of men and $12.6 \%$ of women aged $60-69$, respectively, had diabetes. Of 12909 patients with diabetes, 1415 (11.0\%) had prescriptions for insulin injections, 6049 (46.9\%) had prescriptions for oral antihyperglycemic agents, and $5445(42.2 \%)$ had no diabetes-related prescriptions in the subject-identification year; 1229 $(9.5 \%)$ patients with diabetes were hospitalized due to disease or injury at least once in the quality-reporting year.

\section{Dropout patients and quality indicators}

We observed that 1409 patients (10.9\%) dropped out in total, and among patients taking medication, 474 (6.4\%) dropped out in the quality-reporting year. The crude percentage of quality indicators among the 11500 patients who were adherent to follow-up visits was $84.6 \%$ for $\geq 1 \mathrm{HbAlc}$ tests per year, $49.2 \%$ for $\geq 1 \mathrm{HbA1c}$ tests per 3 months, $32.8 \%$ for eye examinations, and $87.3 \%$ for serum lipid tests, respectively. Those for urine microalbumin excretion tests and serum creatinine tests were $12.3 \%$ and $84.4 \%$, respectively, among the 10087 patients without known kidney and vesicoureteral disorders or renal failure. In all, 1167 patients $(11.6 \%)$ had been tested for both urine microalbumin excretion and serum creatinine. The adjusted percentages of quality indicators among the insulin injection and oral antihyperglycemic agent groups were $76.4 \%$ and $63.4 \%$ for $\geq 1$ HbA1c test per 3 months, $53.5 \%$ and $35.6 \%$ for eye examinations, and $21.4 \%$ and $15.4 \%$ for urine microalbumin excretion tests, respectively (figure 2). ORs for each quality indicator are shown in table 2 . ORs among younger patients tended to be lower for the HbAlc test ( $\geq 1$ per year) and eye examination. Annual HbAlc tests were less common in patients aged $40-49$ years than in patients aged $60-69$ years (OR 0.77 ; $95 \%$ CI 0.66 to 0.91 ; $\mathrm{p}=0.002$ ). The eye examinations rate tended to be lower among men than in women (OR $0.59 ; 95 \%$ CI 0.54 to $0.64 ; \mathrm{p}<0.001)$, among patients aged $40-49$ years than in patients aged $60-69$ years (OR 0.56 ; $95 \%$ CI 0.50 to 0.63 ; $\mathrm{p}<0.001)$, and among patients who had consulted only one clinic (OR $0.60 ; 95 \%$ CI 0.55 to 0.65 ; $\mathrm{p}<0.001$ ). All rates tended to be lower in patients who received no diabetes-related prescriptions than in those who took oral antihyperglycemic agents (as for urine microalbumin excretion test; OR $0.31 ; 95 \%$ CI 0.27 to 0.37 ; $\mathrm{p}<0.001)$.

\section{DISCUSSION}

The principal finding of this study was that $6.4 \%$ of patients with diabetes taking medication dropped out, and that annual test rates were high for the HbAlc, serum creatinine, and serum lipid tests. In contrast, annual eye examination and urine microalbumin excretion test rates were low even among patients with good adherence to follow-up visits despite universal health insurance coverage and generous benefits packages in Japan. A 2-year longitudinal study design that considered dropouts, a large sample of cases, and medication data was the strength of this study compared with previous Japanese studies. ${ }^{27}{ }^{28}$ In addition, we included patients in their 20 s to 40 s who were not adequately represented in earlier studies. 
Table 1 Characteristics of the patients and study population in the subject-identification year (April 2010 to March 2011)

\begin{tabular}{|c|c|c|c|}
\hline & $\begin{array}{l}\text { Patients with diabetes } \\
\text { Number (A) (\%) }\end{array}$ & $\begin{array}{l}\text { Study } \\
\text { population } \\
\text { Number (B) }\end{array}$ & $\begin{array}{l}\text { Proportion of } \\
\text { diabetes } \\
\text { (A)/(B) } \%\end{array}$ \\
\hline Total & $12909(100)$ & 570363 & 2.26 \\
\hline \multicolumn{4}{|l|}{ Age (years) } \\
\hline \multicolumn{4}{|l|}{ Men } \\
\hline Total & $8532(66.1)$ & 320146 & 2.67 \\
\hline 20-29 & 97 & 76187 & 0.13 \\
\hline 30-39 & 641 & 108403 & 0.59 \\
\hline $40-49$ & 2136 & 79252 & 2.70 \\
\hline $50-59$ & 3729 & 44959 & 8.29 \\
\hline $60-69$ & 1929 & 11345 & 17.00 \\
\hline \multicolumn{4}{|l|}{ Women } \\
\hline Total & 4377 (33.9) & 250217 & 1.75 \\
\hline 20-29 & 87 & 52709 & 0.17 \\
\hline 30-39 & 336 & 86179 & 0.39 \\
\hline $40-49$ & 900 & 65771 & 1.37 \\
\hline $50-59$ & 1743 & 35175 & 4.96 \\
\hline $60-69$ & 1311 & 10383 & 12.63 \\
\hline \multicolumn{4}{|l|}{ Prescription* } \\
\hline Insulin injection & $1415(11.0)$ & & \\
\hline Oral antihyperglycemic agents & 6049 (46.9) & & \\
\hline No diabetes-related prescriptions & $5445(42.2)$ & & \\
\hline \multicolumn{4}{|l|}{ Comorbid conditions (ICD-10) } \\
\hline Hyperlipidemia (E78) & 8545 (66.2) & & \\
\hline Hypertension (I10-15) & $7725(59.8)$ & & \\
\hline Ischemic heart disease (I20-25) & $1934(15.0)$ & & \\
\hline Chronic respiratory disease ( J40-47) & $1777(13.8)$ & & \\
\hline Kidney and vesicoureteral disorders (N00-16, 20-39) & 1407 (10.9) & & \\
\hline Cerebral vascular disorder (160-69) & $1252(9.7)$ & & \\
\hline Cancer (C00-99, D00-48) & $833(6.5)$ & & \\
\hline Mental disorders (F20-F39) & $315(2.4)$ & & \\
\hline Renal failure (N17-19) & $314(2.4)$ & & \\
\hline \multicolumn{4}{|l|}{ Types of medical consultation facility } \\
\hline Only one clinic & $6324(49.0)$ & & \\
\hline Others & $6585(51.0)$ & & \\
\hline
\end{tabular}

Routine HbA1c examination ( $\geq 1$ per year) was conducted for most patients, especially in the insulin injection and oral antihyperglycemic agent groups. In these patients, regular testing of HbA1c levels may be required for titrating medications at least once every year. The HbA1c level was checked less frequently in the oral antihyperglycemic agent group than in the insulin injection group. The rates of the serum creatinine and lipid tests were also high. It appeared that both tests were conducted simultaneously with the HbAlc test. Although the JDS guidelines do not recommend an optimal frequency for the serum lipid test, the rate of lipid profile monitoring in Japan was high and comparable to that in the US or European countries. ${ }^{17} 20$

Both annual eye examination and urine microalbumin excretion test rates were lower than the rates for other quality indicators, which was similar to an earlier study. ${ }^{27}$ The eye examination rates in Japan $(35.6 \%$ among patients taking oral antihyperglycemic agents) were much lower than in the US $(73.4 \%)$ and European countries $(74.8 \%) .{ }^{17}{ }^{20}$ As for patient factors, the rates were lower especially among younger patients, which is compatible with a report from the US. ${ }^{39}$ On the other hand, the rate was lower in men than in women, which contradicts a report from Italy. ${ }^{10}$ Although our study population had generally favorable access to healthcare, our findings show that retinopathy is more likely to be overlooked in working-age men than women because male full-time workers with diabetes may not find the time to visit an ophthalmologist owing to the long working hours in Japan. ${ }^{40}$ Rates were also lower among patients who consulted only one clinic in the subject-identification year. In Japan, eye examinations are performed almost exclusively by ophthalmologists; as a result, patients with diabetes usually needed to be referred to another medical facility. ${ }^{26}$ Our findings 


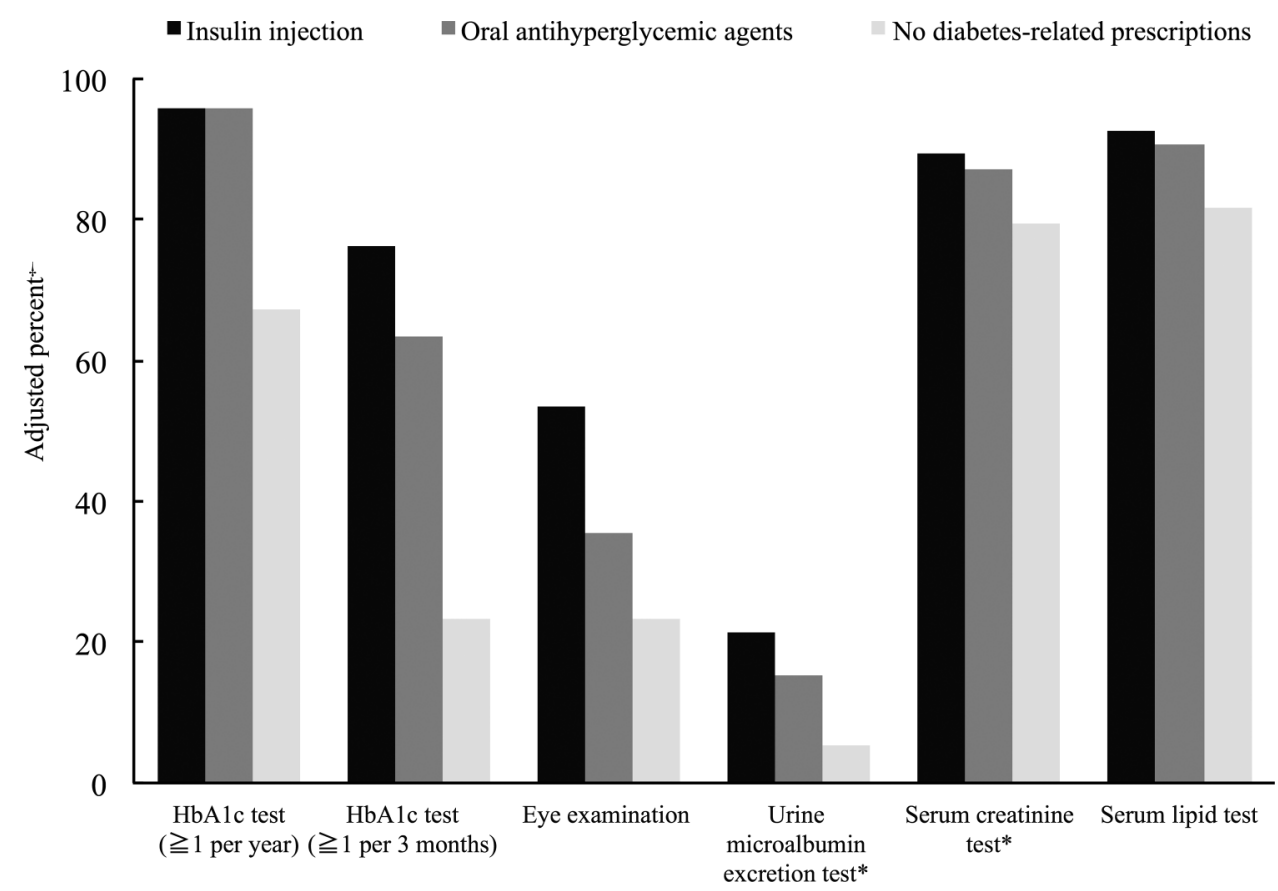

Figure 2 Adjusted annual percentage of quality indicators by prescription. *Excluding patients with kidney and vesicoureteral disorders or renal failure. †Separate multivariate logistic regression models were constructed for each quality indicator. Percentages were calculated from the logistic regression model parameter estimates and are shown here. HbA1c, glycated hemoglobin.

suggest that the referral system between the clinics and ophthalmologists may not work effectively in the primary care setting. Interventions to encourage primary care doctors to use 'the Standard Diabetes Manual' developed by Japanese clinical researchers improved the performance of nephropathy screening, but did not improve retinopathy screening. ${ }^{41}$ Thus, interventions that will facilitate the referral system and enhance retinopathy screening and treatment in patients with diabetes such as written communication systems between primary care physicians and ophthalmologists, are needed. ${ }^{42}$

The annual urine microalbumin excretion test rate in Japan (15.4\% among patients taking oral antihyperglycemic agents) was also much lower than in European countries $(59.4 \%) .^{20}$ Since the serum creatinine screening was optimal, physicians could have been diagnosing nephropathy only from the serum creatinine level, without testing urine microalbumin excretion, which might explain this discrepancy. Early nephropathy may remain undetected owing to such clinical practices. A lack of knowledge among physicians about the efficacy of microalbumin excretion tests or underpayment for these tests may result in this poor performance, despite the JDS guidelines' recommendation of annual urine microalbumin excretion tests. A previous study showed that multifaceted intervention (measuring quality indicators and providing feedback to physicians) was effective for improving the quality of care in primary care settings. ${ }^{38}$ Development and use of effective multifaceted interventions to improve clinical guideline adherence among physicians is key to preventing nephropathy progression in Japan.

Regarding an international comparison of quality of diabetes care, routine screening of retinopathy and nephropathy in Japan appeared to be poorer than in other developed countries, as discussed above. For instance, our findings suggest that quality indicators for nephropathy screening in Japan were much lower than in the UK; $75.6 \%$ of patients with diabetes taking medication were compliant with the UK National Institute for Health and Care Excellence guideline during 20072012. ${ }^{43}$ We suspect that the difference in medical care systems may be one of the reasons for this gap. While patients with diabetes in the UK are taken care of by general practitioners who are financially incentivized to provide a high quality of care (based on the UK's Quality and Outcomes Framework), ${ }^{44} 45$ there are no corresponding systems in Japan; Japanese physicians' pay is not dependent on quality of care provided. In addition, physicians could not afford to spend much time in consultation with outpatients because consultation frequencies are generally high in Japan. ${ }^{25}$ When we consider together these healthcare provider factors, there may be room to reconsider medical care provision systems for chronic disease care in Japan.

In this study, $6.4 \%$ of patients with diabetes taking medication dropped out in the quality-reporting year. As the quality of care and glycemic control would presumably deteriorate in dropouts because of less access to care, encouragement may be required to enhance adherence to follow-ups. The Japan Diabetes Outcome 
Table 2 Adjusted ORs† for process quality indicators by sex, age, prescription, and types of medical consultation facility

\begin{tabular}{|c|c|c|c|c|c|c|c|c|c|}
\hline & \multicolumn{3}{|c|}{ HbA1c test ( $\geq 1$ per year) } & \multicolumn{3}{|c|}{ HbA1c test ( $\geq 1$ per 3 months) } & \multicolumn{3}{|c|}{ Eye examination } \\
\hline & OR & $95 \% \mathrm{Cl}$ & p Value & OR & $95 \% \mathrm{Cl}$ & p Value & OR & $95 \% \mathrm{Cl}$ & p Value \\
\hline \multicolumn{10}{|l|}{ Sex } \\
\hline Men & 1.08 & (0.96 to 1.22$)$ & 0.187 & 0.95 & (0.87 to 1.04$)$ & 0.261 & $0.59^{*}$ & (0.54 to 0.64$)$ & $<0.001$ \\
\hline Women & \multicolumn{3}{|c|}{ Reference } & \multicolumn{3}{|c|}{ Reference } & \multicolumn{3}{|c|}{ Reference } \\
\hline \multicolumn{10}{|l|}{ Age } \\
\hline $20-29$ & $0.60^{*}$ & (0.39 to 0.93$)$ & 0.023 & 1.10 & (0.75 to 1.62$)$ & 0.625 & $0.43^{\star}$ & (0.29 to 0.64$)$ & $<0.001$ \\
\hline $30-39$ & $0.72^{*}$ & (0.57 to 0.91$)$ & 0.005 & 1.05 & (0.88 to 1.26$)$ & 0.587 & $0.53^{*}$ & (0.44 to 0.64$)$ & $<0.001$ \\
\hline $40-49$ & $0.77^{*}$ & (0.66 to 0.91$)$ & 0.002 & 1.05 & (0.93 to 1.19$)$ & 0.406 & $0.56^{*}$ & (0.50 to 0.63$)$ & $<0.001$ \\
\hline $50-59$ & 0.90 & (0.78 to 1.03$)$ & 0.134 & 0.98 & (0.88 to 1.09$)$ & 0.714 & $0.68^{*}$ & (0.61 to 0.75$)$ & $<0.001$ \\
\hline $60-69$ & \multicolumn{3}{|c|}{ Reference } & \multicolumn{3}{|c|}{ Reference } & \multicolumn{3}{|c|}{ Reference } \\
\hline \multicolumn{10}{|l|}{ Prescription } \\
\hline Insulin injection & 1.01 & (0.75 to 1.36$)$ & 0.933 & $1.90^{*}$ & (1.64 to 2.19$)$ & $<0.001$ & $2.15^{\star}$ & (1.89 to 2.44$)$ & $<0.001$ \\
\hline Oral antihyperglycemic agents & \multicolumn{3}{|c|}{ Reference } & \multicolumn{3}{|c|}{ Reference } & \multicolumn{3}{|c|}{ Reference } \\
\hline No diabetes-related prescriptions & $0.09^{*}$ & (0.08 to 0.10$)$ & $<0.001$ & $0.17^{\star}$ & $(0.16$ to 0.19$)$ & $<0.001$ & $0.54^{*}$ & (0.49 to 0.59$)$ & $<0.001$ \\
\hline \multicolumn{10}{|l|}{ Types of consultation medical facility } \\
\hline Only one clinic & $1.14^{*}$ & (1.01 to 1.28$)$ & 0.029 & $0.57^{*}$ & (0.53 to 0.62$)$ & $<0.001$ & $0.60^{*}$ & $(0.55$ to 0.65$)$ & $<0.001$ \\
\hline Others & \multicolumn{3}{|l|}{ Reference } & \multicolumn{3}{|c|}{ Reference } & \multicolumn{3}{|c|}{ Reference } \\
\hline & \multicolumn{3}{|c|}{ Urine microalbumin excretion test } & \multicolumn{3}{|c|}{ Serum creatinine test } & \multicolumn{3}{|c|}{ Serum lipid test } \\
\hline & OR & $95 \% \mathrm{CI}$ & p Value & OR & $95 \% \mathrm{CI}$ & p Value & OR & $95 \% \mathrm{Cl}$ & p Value \\
\hline \multicolumn{10}{|l|}{ Sex } \\
\hline Men & $1.21^{*}$ & (1.06 to 1.39$)$ & 0.005 & 0.92 & (0.81 to 1.03$)$ & 0.153 & 0.90 & (0.80 to 1.02$)$ & 0.107 \\
\hline Women & \multicolumn{3}{|c|}{ Reference } & \multicolumn{3}{|c|}{ Reference } & \multicolumn{3}{|c|}{ Reference } \\
\hline Age & & & & & & & & & \\
\hline $20-29$ & 0.69 & (0.34 to 1.40$)$ & 0.302 & 1.30 & (0.72 to 2.33$)$ & 0.387 & 1.00 & (0.60 to 1.66$)$ & 0.999 \\
\hline 30-39 & 1.17 & (0.90 to 1.52$)$ & 0.233 & 1.07 & (0.83 to 1.37 ) & 0.623 & 0.97 & (0.76 to 1.24$)$ & 0.789 \\
\hline $40-49$ & $1.21^{*}$ & (1.01 to 1.44$)$ & 0.037 & 0.94 & (0.80 to 1.11$)$ & 0.498 & 0.94 & (0.80 to 1.12$)$ & 0.496 \\
\hline $50-59$ & 1.00 & (0.85 to 1.17$)$ & 0.971 & $0.86^{*}$ & (0.75 to 1.00$)$ & 0.043 & 0.92 & (0.80 to 1.06$)$ & 0.256 \\
\hline $60-69$ & Reference & & & Refere & & & Refere & & \\
\hline Prescription & & & & & & & & & \\
\hline Insulin injection & $1.50^{*}$ & $(1.27$ to 1.77$)$ & $<0.001$ & 1.22 & (0.98 to 1.53$)$ & 0.07 & $1.31^{*}$ & (1.03 to 1.65$)$ & 0.026 \\
\hline Oral antihyperglycemic agents & Reference & & & Refere & & & Refere & & \\
\hline No diabetes-related prescriptions & $0.31^{*}$ & $(0.27$ to 0.37$)$ & $<0.001$ & $0.55^{\star}$ & (0.49 to 0.62$)$ & $<0.001$ & $0.45^{*}$ & $(0.40$ to 0.51$)$ & $<0.001$ \\
\hline Type of medical consultation facility & & & & & & & & & \\
\hline Only one clinic & $1.37^{*}$ & $(1.21$ to 1.55$)$ & $<0.001$ & $0.55^{\star}$ & (0.49 to 0.62$)$ & $<0.001$ & $0.63^{*}$ & (0.56 to 0.71$)$ & $<0.001$ \\
\hline Others & Reference & & & Refere & & & Refere & & \\
\hline
\end{tabular}

\section{${ }^{*} \mathrm{p}<0.05$}

†Separate multivariate logistic regression models were constructed for six quality indicators. Sex, age, prescription, medical consultation facility, and nine comorbid conditions (listed in table 1) were included in each multivariate logistic regression model. The references for the ORs in each comorbid condition are the condition-negative subgroups.

HbA1c, glycated hemoglobin. 
Intervention Trial 2 (J-DOIT2) has been launched to reduce dropout and improve clinical outcomes by providing external support to patients and physicians. ${ }^{26} 38$ In addition, all Health Insurance Societies have been required to make health promotion plans using their beneficiaries' health check-up data and health insurance claims data (referred to as 'Data Health Plans') since April 2015 to prevent chronic diseases including diabetic complications and to reduce the economic burden. ${ }^{46}$ For instance, medical consultation encouragement programs for non-consultation or dropouts are included in the 'Data Health Plans'. Although consultation encouragement programs tend to be mainly focused on recent health practice, considering our results, adherence to clinical guidelines and referral systems among physicians (healthcare provider factors) would also need to be improved to enhance the overall quality of diabetes care in Japan.

Some limitations of our study should be noted. First, our study population consisted of workers working in large companies and factories, and their dependents. Since this could induce the 'healthy worker effect', the prevalence of diabetes in this group may have been lower than in the general population. In addition, gender-based differences in retinopathy screening performance may be inferred from the study population. These may affect the generalizability of this study. Second, although we analyzed patient characteristics using prescription data, blood glycemic levels including HbA1c levels for individual patients were unknown. Thus, some patients may have been inappropriately included as patients with type 2 diabetes in this study. This might have led to the underestimation of quality indicators; however, this only applies to patients not on medication. A cross-sectional study reported that the proportion of patients with diabetes treated with diet therapy (no medications) was $19.9 \%$ in 2011; these patients were mainly treated by diabetes specialists in Japan. ${ }^{47}$ Regarding the patients in this study who were not taking medication $(42.2 \%)$, quality indicators of these patients may be underestimated due to misclassification; our definition may include patients with a tentative diagnosis of diabetes. ${ }^{48}$ These data should thus be interpreted with caution. Besides, the urine microalbumin excretion test rate may have been underestimated because patients with overt proteinuria were appropriately tested for urinary protein concentration rather than urine microalbumin concentration. Third, we failed to measure several other process quality indicators recommended by the JDS guidelines, including neuropathy screening, foot care practices, or dental examination because of limited claim data. ${ }^{14}$ Fourth, our definition of dropout may be too strict and lead to misclassification. A previous study defined dropout as over 12 months' non-attendance at the clinic. ${ }^{11}$ However, a routine visit to the clinic or hospital at least every 3 months was considered to be a reasonable frequency in the Japanese healthcare setting. ${ }^{38}$

\section{CONCLUSIONS}

Despite favorable conditions for access to healthcare in Japan, about $6.4 \%$ dropout was observed, and the quality of diabetes care in terms of routine screening of retinopathy and nephropathy appeared to be poorer than in other developed countries, even among patients with good adherence to follow-up visits. The suboptimal quality of care might be attributable to healthcare provider factors that affect retinopathy and nephropathy prevention, as well as patient factors such as low access to retinopathy prevention among working-age men with diabetes. These findings substantially advance previous research in this area as a result of the better study design, large sample size, usage of recorded administrative data, longitudinal analysis with medication data, and availability of international comparisons. Encouraging patient consultations and improvement of physicians' adherence to clinical guidelines and/or referral systems could be key in enhancing the quality of diabetes care.

Contributors HT had full access to all the study data and was responsible for data integrity, the accuracy of the data analysis, and drafting of the manuscript. HT, JT, and YK contributed to the concept and design of the study. JT and YK acquired the data and obtained funding. HT, JT, and TS were responsible for data analysis and interpretation, while HT and JT conducted the statistical analysis. JT, TS, and YK critically reviewed the manuscript. YK also supervised the study and provided administrative, technical, and material support.

Funding This work was supported in part by the Research Funding for Longevity Sciences (23-22) from the National Center for Geriatrics and Gerontology (NCGG), Japan (Kobayashi).

Disclaimer The funder had no role in the design and conduct of the study; collection, management, analysis, and interpretation of the data; preparation, review, or approval of the manuscript; and decision to submit the manuscript for publication.

Competing interests None declared.

Ethics approval The Research Ethics Committee of the Graduate School of Medicine, the University of Tokyo.

Provenance and peer review Not commissioned; externally peer reviewed.

Data sharing statement No additional data are available.

Open Access This is an Open Access article distributed in accordance with the Creative Commons Attribution Non Commercial (CC BY-NC 4.0) license, which permits others to distribute, remix, adapt, build upon this work noncommercially, and license their derivative works on different terms, provided the original work is properly cited and the use is non-commercial. See: http:// creativecommons.org/licenses/by-nc/4.0/

\section{REFERENCES}

1. Guariguata L, Whiting DR, Hambleton I, et al. Global estimates of diabetes prevalence for 2013 and projections for 2035. Diabetes Res Clin Pract 2014;103:137-49.

2. Gaede $\mathrm{P}$, Lund-Andersen $\mathrm{H}$, Parving $\mathrm{HH}$, et al. Effect of a multifactorial intervention on mortality in type 2 diabetes. $N$ Engl J Med 2008;358:580-91.

3. Currie CJ, Peyrot M, Morgan CL, et al. The impact of treatment noncompliance on mortality in people with type 2 diabetes. Diabetes Care 2012;35:1279-84.

4. Morrison F, Shubina M, Turchin A. Encounter frequency and serum glucose level, blood pressure, and cholesterol level control in patients with diabetes mellitus. Arch Intern Med 2011;171:1542-50.

5. Campbell SM, Roland MO, Buetow SA. Defining quality of care. Soc Sci Med 2000;51:1611-25.

6. Nam S, Chesla C, Stotts NA, et al. Barriers to diabetes management: patient and provider factors. Diabetes Res Clin Pract 2011;93:1-9. 
7. Griffin SJ. Lost to follow-up: the problem of defaulters from diabetes clinics. Diabet Med 1998;15(Suppl 3):S14-24.

8. Fosse-Edorh S, Fagot-Campagna A, Detournay B, et al. Impact of socio-economic position on health and quality of care in adults with type 2 diabetes in France: the ENTRED 2007 study. Diabet Med 2015;32:1438-44.

9. Magnan EM, Palta M, Mahoney JE, et al. The relationship of individual comorbid chronic conditions to diabetes care quality. $B M J$ Open Diabetes Res Care 2015;3:e000080.

10. Rossi MC, Cristofaro MR, Gentile S, et al. Sex disparities in the quality of diabetes care: biological and cultural factors may play a different role for different outcomes a cross-sectional observational study from the AMD Annals initiative. Diabetes Care 2013;36:3162-8.

11. Graber AL, Davidson P, Brown AW, et al. Dropout and relapse during diabetes care. Diabetes Care 1992;15:1477-83.

12. Fullerton B, Erler A, Pöhlmann B, et al. Predictors of dropout in the German disease management program for type 2 diabetes. BMC Health Serv Res 2012;12:8.

13. Puder JJ, Keller U. Quality of diabetes care: problem of patient or doctor adherence? Swiss Med Wkly 2003;133:530-4.

14. Tajima N, Noda M, Origasa $\mathrm{H}$, et al. Evidence-based practice guideline for the treatment for diabetes in Japan 2013. Diabetol Int 2015;6:151-87.

15. American Diabetes Association. Standards of medical care in diabetes. Diabetes Care 2016;39(Suppl 1):S1-112.

16. International Diabetes Federation Guideline Development Group. Global guideline for type 2 diabetes. Diabetes Res Clin Pract 2014;104:1-52.

17. Ali MK, Bullard KM, Saaddine JB, et al. Achievement of goals in U.S. diabetes care, 1999-2010. N Engl J Med 2013;368:1613-24.

18. Rivera-Hernandez M, Leyva B, Keohane LM, et al. Quality of care for white and Hispanic Medicare advantage enrollees in the United States and Puerto Rico. JAMA Intern Med 2016;176:787-94.

19. Baillot A, Pelletier C, Dunbar P, et al. Profile of adults with type 2 diabetes and uptake of clinical care best practices: results from the 2011 Survey on Living with Chronic Diseases in Canada-Diabetes component. Diabetes Res Clin Pract 2014;103:11-19.

20. Stone MA, Charpentier G, Doggen K, et al. Quality of care of people with type 2 diabetes in eight European countries: findings from the Guideline Adherence to Enhance Care (GUIDANCE) study. Diabetes Care 2013;36:2628-38.

21. Du Y, Heidemann C, Schaffrath Rosario A, et al. Changes in diabetes care indicators: findings from German National Health Interview and Examination Surveys 1997-1999 and 2008-2011. BMJ Open Diabetes Res Care 2015;3:e000135.

22. Si D, Bailie R, Wang Z, et al. Comparison of diabetes management in five countries for general and indigenous populations: an internet-based review. BMC Health Serv Res 2010;10:169.

23. Flores-Hernández $\mathrm{S}$, Saturno-Hernández PJ, Reyes-Morales $\mathrm{H}$, et al. Quality of diabetes care: the challenges of an increasing epidemic in Mexico. Results from two National Health Surveys (2006 and 2012). PLoS ONE 2015;10:e0133958.

24. Lakkis NA, Mahmassani DM, Hamadeh GN. Diabetes quality of care at a university community in Beirut. J Eval Clin Pract 2015;21:824-30.

25. Ikegami N, Yoo BK, Hashimoto $\mathrm{H}$, et al. Japanese universal health coverage: evolution, achievements, and challenges. Lancet 2011;378:1106-15.

26. Imamura S. Countermeasures against Diabetes in Japan and Efforts Made by the Japan Medical Association. Japan Med Assoc J 2010;53:3-7.

27. Tomio J, Toyokawa S, Tanihara S, et al. Quality of care for diabetes patients using National Health Insurance claims data in Japan. $J$ Eval Clin Pract 2010;16:1164-9.

28. Kabeya Y, Maki K, Mari O, et al. Quality control in diabetes care using a method of statistical process control. Diabetol Int 2014;5:219-28.
29. Kimura S, Sato T, Ikeda S, et al. Development of a database of health insurance claims: standardization of disease classifications and anonymous record linkage. J Epidemiol 2010;20:413-19.

30. Ministry of Health Labour and Welfare. Health and Medical Services. 2012. http://www.mhlw.go.jp/english/wp/wp-hw6/d//02e.pdf (accessed 28 Jul 2016).

31. Goto A, Goto M, Terauchi $Y$, et al. Association between severe hypoglycemia and cardiovascular disease risk in Japanese patients with type 2 diabetes. J Am Heart Assoc 2016;5:e002875.

32. Tsujimura Y, Takahashi Y, Ishizaki T, et al. Predictors of hyperglycaemic individuals who do not follow up with physicians after screening in Japan: a cohort study. Diabetes Res Clin Pract 2014;105:176-84.

33. Heianza Y, Suzuki A, Fujihara K, et al. Impact on short-term glycaemic control of initiating diabetes care versus leaving diabetes untreated among individuals with newly screening-detected diabetes in Japan. J Epidemiol Community Health 2014;68:1189-95.

34. Fukushima K, Mizuoka S, Yamamoto S, et al. Patient cost sharing and medical expenditures for the Elderly. $J$ Health Econ 2016;45:115-30.

35. Takizawa $\mathrm{O}$, Urushihara $\mathrm{H}$, Tanaka $\mathrm{S}$, et al. Price difference as a predictor of the selection between brand name and generic statins in Japan. Health Policy 2015;119:612-19.

36. Ohisa M, Kimura Y, Matsuo J, et al. Estimated numbers of patients with liver disease related to hepatitis $B$ or $C$ virus infection based on the database reconstructed from medical claims from 2008 to 2010 in Japan. Hepatol Res 2015;45:1228-40.

37. European Pharmaceutical Market Research Association. EphMRA Anatomical Classification Guidelines. 2015. http://www.ephmra.org/ user_uploads/atcguidelines2015final.pdf (accessed 28 Jul 2016).

38. Hayashino $\mathrm{Y}$, Suzuki $\mathrm{H}$, Yamazaki $\mathrm{K}$, et al. A cluster randomized trial on the effect of a multifaceted intervention improved the technical quality of diabetes care by primary care physicians: the Japan Diabetes Outcome Intervention Trial-2 (J-DOIT2). Diabet Med 2016;33:599-608.

39. Villarroel MA, Vahratian A, Ward BW. Health care utilization among U.S. adults with diagnosed diabetes, 2013. NCHS Data Brief 2015;183:1-8.

40. Genda Y, Kuroda S, Ohta S. Does downsizing take a toll on retained staff? An analysis of increased working hours in the early 2000 s in Japan. J Jpn Int Econ 2015;36:1-24.

41. Noto $\mathrm{H}$, Tanizawa $\mathrm{Y}$, Aizawa $\mathrm{T}$, et al. Cluster-randomized trial to improve the quality of diabetes management: the study for the efficacy assessment of the Standard Diabetes Manual (SEAS-DM). J Diabetes Investig 2016;7:539-43.

42. Storey PP, Murchison AP, Pizzi LT, et al. Impact of physician communication on diabetic eye examination adherence: results from a retrospective cohort analysis. Retina (Philadelphia, $\mathrm{Pa}$ ) 2016;36:20-7.

43. Liang $\mathrm{H}$, Kennedy $\mathrm{C}$, Manne $\mathrm{S}$, et al. Monitoring for proteinuria in patients with type 2 diabetes mellitus. BMJ Open Diabetes Res Care 2015;3:e000071.

44. Calvert M, Shankar A, McManus RJ, et al. Effect of the quality and outcomes framework on diabetes care in the United Kingdom: retrospective cohort study. BMJ 2009;338:b1870.

45. Kontopantelis E, Reeves D, Valderas JM, et al. Recorded quality of primary care for patients with diabetes in England before and after the introduction of a financial incentive scheme: a longitudinal observational study. BMJ Qual Saf 2013;22:53-64.

46. Ministry of Health Labour and Welfare. Guide of the Data Health Plans. http://www.mhlw.go.jp/file/06-Seisakujouhou-12400000Hokenkyoku/0000069358.pdf (accessed 28 Jul 2016).

47. Oishi M, Yamazaki K, Okuguchi F, et al. Changes in oral antidiabetic prescriptions and improved glycemic control during the years 20022011 in Japan (JDDM32). J Diabetes Investig 2014;5:581-7.

48. Tanihara S, Okamoto E, Une H. A statistical analysis of 'rule-out' diagnoses in outpatient health insurance claims in Japan. J Eval Clin Pract 2011;17:1070-4. 